\title{
Towards a Density-Based Description of Chemical Bonds and Noncovalent Interactions with Pauli Energy
}

\author{
Shujing Zhong, ${ }^{1}$ Xin He, ${ }^{1}$ Siyuan Liu, ${ }^{1}$ Bin Wang, ${ }^{1,2}$ Tian Lu, ${ }^{3}$ \\ Chunying Rong, ${ }^{1}$ and Shubin $\mathrm{Liu}^{4,5^{*}}$ \\ ${ }^{1}$ Key Laboratory of Chemical Biology and Traditional Chinese Medicine Research \\ (Ministry of Education of China), Hunan Normal University, \\ Changsha, Hunan 410081, P.R. China \\ E-mail: rongchunying@aliyun.com \\ ${ }^{2}$ Eenheid Algemene Chemie, Vrije Universiteit Brussel, Faculteit Wetenschappen, Pleinlaan 2, \\ 1050 Brussels, Belgium \\ ${ }^{3}$ Beijing Kein Research Center for Natural Sciences, Beijing 100022, P. R. China \\ ${ }^{5}$ Research Computing Center, University of North Carolina, Chapel Hill, North Carolina 27599- \\ 3420, USA. E-mail: shubin@email.unc.edu \\ ${ }^{6}$ Department of Chemistry, University of North Carolina, Chapel Hill, North Carolina, 27599- \\ 3290, USA
}

\begin{abstract}
Chemical bonds and noncovalent interactions are extraordinarily important concepts in chemistry and beyond. Using density-based quantities to describe them has a long history in the literature, yet none can satisfactorily describe the entire spectrum of interactions from strong chemical bonds to weak van der Waals forces. In this work, employing Pauli energy as the theoretical foundation, we fill in that knowledge gap. Our results show that the newly established density-based index can describe single and multiple covalent bonds, ionic bonds, metallic bonds, and different kinds of noncovalent interactions, all with unique and readily identifiable signature shapes. Two new descriptors, NBI (nonbonding and bonding identification) index and USI (ultra-strong interaction) index, have been introduced in this work. Together with $\mathrm{NCl}$ (noncovalent interaction) and $\mathrm{SCl}$ (strong covalent interaction) indexes already available in the literature, a density-based description of both chemical bonds and noncovalent interactions is accomplished.
\end{abstract}


Essential to maintain stable three-dimensional structures and appreciate various physiochemical properties for all molecules including proteins and nucleic acids, chemical bonds and noncovalent interactions are exceptionally important concepts in chemistry. Chemical bonds as strong interactions between two atoms of a molecule can often be categorized into ionic, covalent, metallic, coordinate bonds, etc. Covalent bonds can be single and multiple bonds with a single bond corresponding to sharing one pair of electrons and a multiple bond sharing multiple pairs, respectively. Noncovalent interactions, which are often weak, usually include electrostatic, $\pi$-stacking, van der Waals forces, hydrophobic effects, etc. Traditionally, chemical bonding can be understood through the introduction of orbitals, from either valence bond theory or molecular orbital theory, whereas noncovalent interactions may be explained in terms of charge, dipole, and dispersion effects.

As density functional theory (DFT) became mature in the past few decades, ${ }^{1}$ efforts using density-based quantities to comprehend both strong and weak interactions are available in the literature. ${ }^{2-5}$ Examples include AIM (atoms in molecules), ${ }^{2}$ ELF (electron localization function), ${ }^{3-5}$ and $\mathrm{NCl}$ (noncovalent interaction) $)^{6,7}$ analyses. However, one issue with these analyses is that they are empirical and phenomenological without a solid foundation for their general validity and applicability. To overcome the issue, earlier, we proposed the densitybased $\mathrm{SCl}$ (strong covalent interaction) index ${ }^{8}$ to identify the multiplicity of strong covalent bonds between two atoms in molecules using the characteristic isosurface shape. For double, triple, quadruple, and quintuple covalent bonds, for example, the signature $\mathrm{SCl}$ isosurface shape in the center of the bonds is like dumbbell, donut (torus), four beads, and two corn beans, respectively. ${ }^{8-10}$ The theoretical basis of the $\mathrm{SCl}$ index is that strong covalent interactions 
are dominated by electron correlations originated from the Pauli Exclusion Principle, which can be quantified by the Pauli energy. ${ }^{11}$ The downside of this index is that it is not applicable to other bonding types such as single covalent bond, ionic, metallic, and noncovalent interactions.

In this study, on the same theoretical foundation, we extend our previous work to cover other types of bonding as well as nonbonding interactions. We still make use of Pauli energy as the descriptor. The new index is applicable to covalent, ionic, and metallic bonds, plus noncovalent interactions including electrostatic, dipole, van der Waals forces. Different from other density-based indexes available in the literature such as $\mathrm{NCl}$, our approach is noumenonic and down to the bottom of the matter, which is due to the existence of the Pauli Exclusion Principle leading to the redistribution of the electron density between atoms in molecules when different kinds of strong and weak bonding interactions take place. Also, in the same spirit as $\mathrm{NCl}$, we will unveil a new density-based dimensionless descriptor, called the USI (ultra-strong interaction) index, which, as will be illustrated later, can be employed to identify quadruple, quintuple, and sextuple covalent bonds.

Our working hypothesis is that the interatomic areas with bonding interactions occurring, whether they are strong or weak, are dominated by electron correlations originated from the Pauli Exclusion Principle. In other words, even though other interactions such as kinetic and electrostatic components do play important roles in bringing atoms together, their contributions are largely offset and canceled out by one another. The validity and applicability of this hypothesis have been previously demonstrated by the $\mathrm{SCl}$ index for multiple covalent bonds with the bond order equal to two, three, four, five, and even six..$^{8-10}$ Here, we 
hypothesize that the same principle should also be applicable to other types of chemical bonding and noncovalent interactions so that its coverage to the entire spectrum of bonding and nonbonding interactions is made possible.

In $\mathrm{DFT}^{1}$ the contribution from the Pauli Exclusion Principle can be quantified by Pauli energy $E_{p}{ }^{11}$

$$
E_{P}[\rho] \equiv \int \varepsilon_{P}(\mathbf{r}) d \mathbf{r}=T_{S}[\rho]-T_{W}[\rho] \equiv \int\left(\tau(\mathbf{r})-\tau_{W}(\mathbf{r})\right) d \mathbf{r},
$$

With

$$
\mathrm{T}_{\mathrm{W}}[\rho]=\frac{1}{8} \int \frac{|\nabla \rho(\mathbf{r})|^{2}}{\rho(\mathbf{r})} d \mathbf{r}
$$

where $\rho$ is the electron density, $\nabla \rho(\mathbf{r})$ is the electron density gradient, $E_{P}, T_{S}$, and $T_{W}$ stand for the Pauli energy, total non-interacting kinetic energy, and Weizsäcker kinetic energy, ${ }^{12}$ respectively, and $\varepsilon_{\mathrm{P}}, \tau$, and $\tau_{\mathrm{W}}$ represent their corresponding local energy density. Pauli energy is the energetic contribution from the Pauli principle to the kinetic energy. ${ }^{13-15}$ There is also a contribution to the potential energy, i.e., the exchange-correlation energy, but its magnitude is much smaller.

To convert the Pauli energy to be dimensionless, we define a local function $\zeta(\mathbf{r})$, the scaled Pauli energy density, ${ }^{8}$

$$
\zeta(\mathbf{r}) \equiv \frac{\varepsilon_{P}(\mathbf{r})}{\tau_{T F}(\mathbf{r})}=\frac{\tau(\mathbf{r})-\tau_{W}(\mathbf{r})}{\tau_{T F}(\mathbf{r})}
$$

where $\tau_{\mathrm{TF}}(\mathbf{r})=3\left(6 \pi^{2}\right)^{2 / 3} \rho(\mathbf{r})^{5 / 3} / 5$ denotes the Thomas-Fermi kinetic energy density, which is exact for homogeneous electron gas. Since $\zeta(\mathbf{r})$ diverges asymptotically because $\tau_{T F}(\mathbf{r})$ decays much faster than $\varepsilon_{P}(\mathbf{r})$, we define the $\mathrm{SCl}$ index as the reciprocal of $\zeta(\mathbf{r})$,

$$
\mathrm{SCl}=\frac{1}{\zeta(\mathbf{r})}=\frac{\tau_{T F}(\mathbf{r})}{\tau(\mathbf{r})-\tau_{W}(\mathbf{r})}
$$

In the literature, ${ }^{3}$ Becke and coworker employed $\zeta(\mathbf{r})$ to define ELF, 


$$
E L F=\frac{1}{1+\zeta^{2}(\mathbf{r})}
$$

which measures the likelihood of finding an electron with the same spin in the neighborhood space of a reference electron located at a given point $r$. Notice that even though they have vastly different purposes, both $\mathrm{SCl}$ and ELF indexes employ the same scaled Pauli energy, Eq. (3). The only difference between Eq. (4) and Eq. (5) is how they are presented. SCl is a straightforward measurement of the contribution from the Pauli Exclusion Principle, whereas ELF was to identify regions where parallel electrons are localized. Our previous results ${ }^{8}$ show that both $\mathrm{SCl}$ and ELF yield exceedingly similar results when they are employed to identify strong covalent interactions. Their signature isosurface shapes are the same.

One drawback of the $\mathrm{SCl}$ index in Eq. (4) is that for one and two electron systems, where $\tau(\mathbf{r})$ and $\tau_{\mathrm{W}}(\mathbf{r})$ are identical, so $\zeta(\mathbf{r})=0$ and Eq. (4) diverges. For strong covalent interactions where two or more pairs of electrons are shared, this divergence behavior should not be a big issue. However, since we intend to extend its coverage to include other forms of chemical bonding and noncovalent interactions, the divergence problem of Eq. (4) is no longer acceptable. To overcome this, we propose a new scaled Pauli energy, which we call NBI (nonbonding and bonding identification) index,

$$
\mathrm{NBI} \equiv \frac{\varepsilon_{P}(\mathbf{r})}{\tau_{W}(\mathbf{r})}=\frac{\tau(\mathbf{r})-\tau_{W}(\mathbf{r})}{\tau_{W}(\mathbf{r})} .
$$

Instead of using the Thomas-Fermi energy as the reference, we make use of the Weizsäcker kinetic energy itself. The reason that we call it BNI index, as will be shown below, is that this new function can identify both covalent and noncovalent interactions. Why could this new form be a better choice than Eq. (3)? Replacing $\tau_{\mathrm{HF}}(\mathbf{r})$ with $\tau_{\mathrm{W}}(\mathbf{r})$ in the denominator not only makes the quantity equally dimensionless, but it also avoids the divergence problem because 
$\tau_{W}(\mathbf{r})$ is well behaved asymptotically. Also, expressing Eq. (6) in the format of the relative change of the non-interacting kinetic energy density with respect to the Weizsäcker kinetic energy makes better sense mathematically. In addition, our earlier study proved that the Weizsäcker kinetic energy can be employed to quantify the steric effect, ${ }^{13}$ and it could be applied to appreciate numerous chemical processes and reactivity properties ${ }^{14-18}$ including stereoselectivity. ${ }^{19-22}$ In this regard, Eq. (6) excludes the steric effect from the consideration when we discuss bonding interactions, which makes a lot of sense from the viewpoint of physical chemistry. Since $\tau_{W}(\mathbf{r})$ is a well-behaved local function, we do not even have to use its reciprocal form, like the $\mathrm{SCl}$ index in Eq. (4). The only disadvantage of Eq. (6) is that for one and two electron systems, BNI vanishes, so Eq. (6) is not applicable to simplest systems such as $\mathrm{H}_{2}{ }^{+}$ and $\mathrm{H}_{2}$. This limitation originates from the Pauli energy itself. For these systems, we can resort to other density-based indexes like $\mathrm{NCl}$ and the new index, USI, introduced below.

$\mathrm{NCl}$ as a tool to visualize and identify noncovalent interactions introduced in the literature is based on another dimensionless density-based quantity, the reduced density gradient, ${ }^{6,7}$

$$
\mathrm{NCl} \equiv \frac{|\nabla \rho(\mathbf{r})|}{\rho^{4 / 3}(\mathbf{r})}
$$

For simplicity, we neglected the coefficient in the front. From the dimensionality analysis viewpoint as has been extensively discussed elsewhere, ${ }^{23-25}$ the following dimensionless function is equally worthy of our attention,

$$
\mathrm{USI} \equiv \frac{\nabla^{2} \rho(\mathbf{r})}{\rho^{5 / 3}(\mathbf{r})}
$$

where $\nabla^{2} \rho(\mathbf{r})$ is the Laplacian of the electron density. The reason why we call it the USI (ultrastrong interaction) index is because, as our results will show below, it is a robust descriptor for describing systems with ultra-strong interactions with quadruple, quintuple, and sextuple 
covalent bonds. We note in passing that, same as Eq. (3), Eqs. (7) and (8) also have divergence issues because the denominator decays too fast. However, as our studies found, employing their reciprocal will yield the exact same signature shapes when different isosurface values are used. For this reason, we do not consider alternative local functions stemmed from them.

We first present our comparative studies for a few bimetallic complexes with quadruple and quintuple bonds made of the ligands shown in Scheme S1. In Table S1, the SCl index result for the seven complexes all indicates that there exists the signature four-bead shape between the two metal atoms, suggesting that in these systems the metal-metal bond is quadruple. This result is confirmed by the four LMOs illustrated in the Table together with the result from Mayer bond order analysis. ${ }^{26}$ The same is true for eight quintuple systems in Table S2, where $\mathrm{SCl}$ index exhibits two corn-bean characteristic isosurface shape for all of them, which is verified by the results from LMO and Mayer bond order analyses.

Figure 1 shows the comparison of $\mathrm{SCl}$ and $\mathrm{BNI}$ results for four molecules each with a double bond. The double bonds involved include $\mathrm{C}=\mathrm{C}, \mathrm{Si}=\mathrm{Si}, \mathrm{S}=\mathrm{S}$ and $\mathrm{C}=\mathrm{O}$. For the $\mathrm{SCl}$ index, the signature shape between the two bonded atoms is like a dumbbell or its deformed shape, and for the BNI index, the signature shape is like a propeller blade. Notice that these two signature shapes are perpendicular to each other. Despite that, the signature shapes are identical across different systems with different types of double bonds.

Illustrated in Fig. 2 are fours examples of comparisons between $\mathrm{SCl}$ and $\mathrm{BNI}$ results for systems with different kinds of triple bonds, $\mathrm{C} \equiv \mathrm{C}, \mathrm{Si} \equiv \mathrm{Si}, \mathrm{N} \equiv \mathrm{N}$ and $\mathrm{Th} \equiv \mathrm{Th}$. The signature isosurface shape between bonded atoms for $\mathrm{SCl}$ is like a donut or torus, whereas that for BNI 
is like a cylinder or its compressed form, a thin disc. Again, these characteristic shapes are identical across different systems with different triple bond types.

Besides strong covalent interactions, using the BNI index we can identify ionic and metallic bonds with different signature isosurface shapes, as displayed in Fig. 3 with six illustrative examples. Figures $3 a-3 c$ are ionic bonds for diatoms $\mathrm{LiF}, \mathrm{NaCl}$, and $\mathrm{NaH}$, with the BNI signature shape between the two bonded atoms like a bowl facing the cationic atom. For the metallic bond in Figs. $3 \mathrm{~d}-3 \mathrm{f}$ for $\mathrm{Li}_{2}, \mathrm{Na}_{2}$ and $\mathrm{LiNa}$, the BNI signature shape is like two oppositely stacked bowls with the open sides facing both metal atoms.

The BNI index can also describe single covalent bonds. Shown in Fig. 4 are eight examples of molecules with single covalent bonds including $\mathrm{F}-\mathrm{F}, \mathrm{Cl}-\mathrm{Cl}, \mathrm{F}-\mathrm{Cl}, \mathrm{O}-\mathrm{O}, \mathrm{O}-\mathrm{N}, \mathrm{C}-\mathrm{C}$, Si-Si and $\mathrm{C}-\mathrm{O}$ bonds. As can be seen from the Figure, the signature shape is like a grinding wheel or its deformed form with handlebars connected to both bonded atoms. Again, as shown in the Figure, the same signature shape is observed for different molecules with different types of single covalent bonds.

How about species with noncovalent interactions? Elucidated in Fig. 5 are a few such systems with dispersion interactions ( $\mathrm{He}_{2}$ in Fig. $5 \mathrm{a}, \mathrm{Ar}_{2}$ in Fig. 5b, methane dimer in Fig. 5g), charge ( $\mathrm{Na}^{+} \mathrm{He}$ in Fig. $5 \mathrm{c}$ ) and dipole ( $\mathrm{HF}-\mathrm{He}$ in Fig. $\left.5 \mathrm{~d}\right)$ interactions, and hydrogen bonding (water dimer in Fig. 5e and HF dimer in Fig. 5f). The signature isosurface characterizing these noncovalent interactions is a disc, which is disjoint with the two interacting atoms. This disjointness feature of the signature shape differentiates it from that of the single covalent bond. Again, for all the examples shown in the Figure, the signature shape is always the same. Notice that this feature is markedly like the result from the $\mathrm{NCl}$ index, ${ }^{6,7}$ Eq. (7). To manifest 
this viewpoint, exhibited in Fig. 6 are $\mathrm{NCl}$ results for the same seven systems. As can be seen from the Figure, the common feature of these isosurfaces is also the disc disjoint with the interacting atoms. Compared with those from $\mathrm{NBI}$, the sizes of the discs from $\mathrm{NCl}$ are discernibly smaller though.

While Eqs. (6) and (7) can both identify exceedingly weak interactions between atoms, our study unveils that Eq. (8) goes to the other extreme, i.e., for describing ultra-strong interactions. Shown in Fig. 7 are ten exemplary systems with quadruple (Figs. 7a - 7f), quintuple (Figs. 7g and 7h), and sextuple (Figs. 7i and 7j) bonds. We found that (i) the signature isosurface is the same for the compounds with the same bond multiplicity and (ii) these signature shapes are also the same as those of the $\mathrm{SCl}$ index. ${ }^{8-10}$ That is, for quadruple bonds, the signature shape is four beads, for quintuple bonds, it is two corn beans, and for sextuple bonds, it is two parallel tori or donuts. These results show that USI can obtain consistent results with $\mathrm{SCl}$. For the two examples with the sextuple bond order, we have confirmed our results with LMO and Mayer bond order analyses, whose results are contained in Table S3.

Put together, our results from the present study establish that, through the density-based SCl index in Eq. (4) and the density-based BNI index in Eq. (6), where the Pauli energy was both employed as the root cause governing the interatomic interactions, we can identify and describe the entire spectrum of interactions between atoms in molecules from chemical bonding to noncovalent interactions. This description can be supplemented by two other density-based quantities, the $\mathrm{NCl}$ index, Eq. (7), for weak interactions and the USI index, Eq. (8), for ultra-strong interactions. 
Traditionally, chemical bonds can adequately be appreciated through the orbital picture, either from valence bond theory or molecular orbital theory, but for noncovalent interactions, orbitals are no longer applicable. Using density-based quantities to describe both chemical bonds and noncovalent interactions has a long history in the literature including AIM, ELF and $\mathrm{NCl}$, yet a solid foundation has never been laid down before. This is the precise reason why a satisfactory description for the entire spectrum of interatomic interactions from strong chemical bonds to weak van der Waals interactions is lacking. This work intends to fill in that gap. With the Pauli Exclusion Principle as the root cause, two proposed indexes, SCl and NBI, work together to get the job done. They are also supplemented by two other density-based indexes, $\mathrm{NCl}$ and $\mathrm{USI}$, for the two extreme cases.

One issue with the density-based approach is that it cannot pinpoint the nature of a chemical bond, whether it is $\sigma$, or $\pi$, or $\delta$ type. What we know is only the bond multiplicity and the kind of bonding/nonbonding interactions. Indeed, other bond types can also contribute to make a double or triple bond. Shown in Fig. 8 are six examples from the wellknown 3c-2e (three-center two-electron) bond. Using the signature isosurface shape, we know that B-B and Al-Al bonds in Figs. 8a-8d are double bonds (with the propeller blade like isosurface) contributed from two 3c-2e bonds, and those in Figs. 8 e and $8 \mathrm{f}$ are triple bonds (with the deformed cylinder like shape) consisting of three 3c-2e bonds. Conventionally, we never thought of the B-B or Al-Al bonding in that manner because there are bridging hydrogen atoms between the two boron or aluminum atoms. It does not make sense from the orbital perspective to think that a bridged B-B or Al-Al bond can have a certain degree of a double or triple bond. Nevertheless, from the density-based perspective, as shown in Fig. 8, the overall 
pattern of these bonds is consistent, apparent and distinct. This perspective does provide with us new insight and novel way to look into the matter for this once quite controversial system in chemistry. ${ }^{27}$ What these examples also tell us in a nutshell is that both orbital-based and density-based descriptions of strong and weak interactions between atoms in molecules should be complementary to each other. They should never be mutually exclusive.

Also shown in Fig. 8 are two examples of systems with special bonding types, one with the halogen bond ${ }^{28}$ and the other with the chalcogen bond. ${ }^{29}$ As can be seen from their signature shapes, which are different from both the ionic bond in Fig. 3 and noncovalent interactions in Fig. 5, our results validate that they indeed possess special features and thus can be categorized as different bonding types.

In summary, in this work, a density-based approach to describe interatomic interactions spanning the entire spectrum from chemical bonds to noncovalent interactions have been proposed and established. Its theoretical foundation is the Pauli Exclusion Principle quantified by the Pauli energy. The BNI index introduced in this work can describe single and multiple covalent bonds, ionic bonds, metallic bonds, and different kinds of noncovalent interactions including halogen and chalcogen bonds, all with unique signature isosurface shapes. Together with previously established $\mathrm{NCl}$ and $\mathrm{SCl}$ indexes and the newly introduced index, USI for ultrastrong interactions, in this work, a density-based approach to describe chemical bonds and noncovalent interactions is now available. Numerous examples to illustrate the validity and applicability of our approach have been presented in this work. We finally discussed the downside of the density-based approach as well as the relationship with the orbital-based approach. It is anticipated that more applications will be unveiled in the future. 
Supporting Information Available: Computational details, comparison results of SCI with LMO and Mayer bond order results for systems with quadruple, quintuple and sextuple bonds discussed in this work are available free of charge via the Internet at http://pubs.acs.org.

\section{ACKNOWLEDGMENTS}

We are grateful to Dr. Dongbo Zhao of Yunnan University for helpful discussion. SJZ and CYR acknowledge support from the science and technology innovation Program of Hunan Province (2021RC1003).

\section{References}

1. Parr, R. G.; Yang, W. Density-Functional Theory of Atoms and Molecules. Oxford University Press, USA, 1989.

2. R. F. W. Bader, Atoms in Molecules - A Quantum Theory, Oxford University Press, Oxford, U.K., 1990.

3. Becke, A.D.; Edgecombe, K.E. A Simple Measure of Electron Localization in Atomic and Molecular Systems. J. Chem. Phys. 1990, 92, 5397-5403.

4. Savin, A.; Nesper, R.; Wengert, S.; Fassler, T. F. ELF: The Electron Localization Function. Angew. Chem., Int. Ed. Engl. 1997, 36, 1808- 1832.

5. Savin, A.; Jepsen, O.; Flad, J.; Andersen, O.K.; Preuss, H.; von Schering, H.G. Electron Localization in Solid-State Structures of the Elements: The Diamond Structure. Angew. Chem. Int. Ed. Engl. 1992, 31, 187-188.

6. Johnson, E. R.; Keinan, S.; Mori-Sánchez, P.; Contreras-García, J.; Cohen, A. J.; Yang, W. Revealing Noncovalent Interactions. J. Am. Chem. Soc. 2010, 132, 6498-6506.

7. Contrearas-Garcia, J.; Johnson, E.R.; Keinan, S.; Chaudret, R.; Piquemal, J.-P.; Beratan, D.N. Yang, W. NCIPLOT: A Program for Plotting Noncovalent Interaction Regions. J. Chem. Theory Comput. 2011, 7, 625 - 632. 
8. Liu, S.B.; Rong, C.; Lu, T.; Hu. H. Identifying Strong Covalent Interactions with Pauli Energy. J. Phys. Chem. A 2018, 122, 3087 - 3095.

9. Huang, Y.; Liu, L.; Rong, C.; Lu, T.; Ayers, P.W.; Liu, S.B. SCl: A Robust and Reliable Density-Based descriptor to Determine Multiple Covalent Bond Orders. J. Mol. Modeling 2018, 24, 213.

10. Rong, C.; Wang, B.; Zhao, D.; Liu, S.B. Information-Theoretic Approach in Density Functional Theory and its Recent Applications to Chemical Problems. Wiley Interdiscip. Rev.: Comput. Mol. Sci. 2020, 10, e1461.

11. Holas, A.; March, N. H. Construction of the Pauli Potential, Pauli energy, and Effective Potential from the Electron Density. Phys. Rev. A: At., Mol., Opt. Phys. 1991, 44, 55215536.

12. von Weizsäcker, C. F. Zur Theorie der Kernmassen. Eur. Phys. J. A 1935, 96, 431- 458.

13. Liu, S. B. Steric Effect: A Quantitative Description from Density Functional Theory. J. Chem. Phys. 2007, 126, 244103.

14. Liu, S.B. Origin and Nature of Bond Rotation Barriers: A Unified View, J. Phys. Chem. A 2013, $17,962-965$.

15. Liu, S.B.; Hu, H.; Pedersen, L.G. Steric, Quantum, and Electrostatic Effects on SN2 Reaction Barriers in Gas Phase, J. Phys. Chem. A 2010, 114, 5913-5918.

16. Liu, S.B.; Schauer, C.K. Origin of Molecular Conformational Stability: Perspectives from Molecular Orbital Interactions and Density Functional Reactivity Theory, J. Chem. Phys. 2015, $142,054107$.

17. Li, M; He, X.; Chen, J.; Wang, B.; Liu, S.B.; Rong, C. Density Functional Theory and InformationTheoretic Approach Study on the Origin of Homochirality in Helix Structures. J. Phys. Chem. A $2021,125,1269-1278$.

18. Liu, S.B. Principle of Chirality Hierarchy in Three-Blade Propeller Systems, J. Phys. Chem. Lett. 2021, 12, 8720-8725.

19. Liu, S. B.; Rong, C. Y.; Lu, T. Electronic Forces as Descriptors of Nucleophilic and Electrophilic Regioselectivity and Stereoselectivity. Phys. Chem. Chem. Phys. 2017, 19, 1496- 1503.

20. Liu, S. B.; Liu, L. H.; Yu, D. H.; Rong, C. Y.; Lu, T. Steric Charge. Phys. Chem. Chem. Phys. 2018, $20,1408-1420$. 
21. Liu, S. B. Information-Theoretic Approach in Density Functional Reactivity Theory. Acta Phys. Chim. Sin. 2016, 32, 98- 118.

22. Liu, S.B. Conceptual Density Functional Theory and Some Recent Developments. Acta Phys.-Chim. Sin. 2009, 25, 590- 600.

23. Liu, S.B.; Parr, R.G. Expansions of the Correlation-Energy Density Functional E(c)[rho] and its Kinetic-Energy Component Tc[rho] in terms of Homogeneous Functionals. Phys. Rev. A. $1996,53,2211-2219$.

24. Liu, S.B.; Parr, R.G. Atomic Correlation Energy from the Electron Density at the Nucleus. J. Phys. Chem. A 2007, 111, $10422-10425$.

25. Liu, S. B.; Morrison, R. C.; Parr, R. G. Approximate Scaling Properties of the Density Functional Theory Tc for Atoms J. Chem. Phys. 2006, 125, 174109.

26. Bridgeman, A.J.; Cavigliasso, G.; Ireland, L.R.; Rothery, J. The Mayer Bond Order as a Tool in Inorganic Chemistry. J. Chem. Soc. Dalton Trans. 2001, 2001, 2095 - 2108.

27. Laszlo, P. A Diborane Story. Angew. Chem. Int. Ed. 2000, 39, 2071-2072.

28. Cavallo, G.; Metrangolo, P.; Milani,R.; Pilati, T.; Priimagi, A.; Resnati, G.; Terraneo, G. The Halogen Bond. Chem. Rev. 2016, 116, 2478 - 2601.

29. Pascoe, D.J.; Ling, K. B.; Cockroft, S. L. The Origin of Chalcogen-Bonding Interactions. J. Am. Chem. Soc. 2017, 139, 15160-15167. 


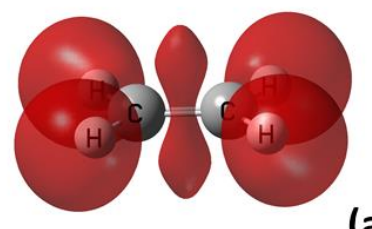

(a)

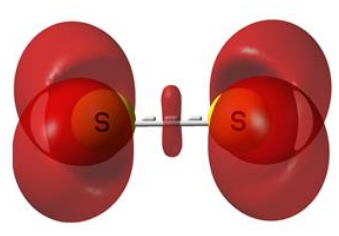

(c)
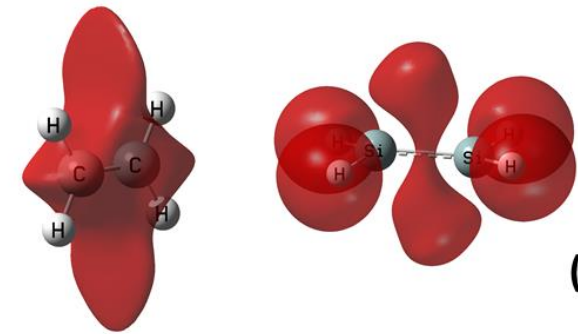

(b)
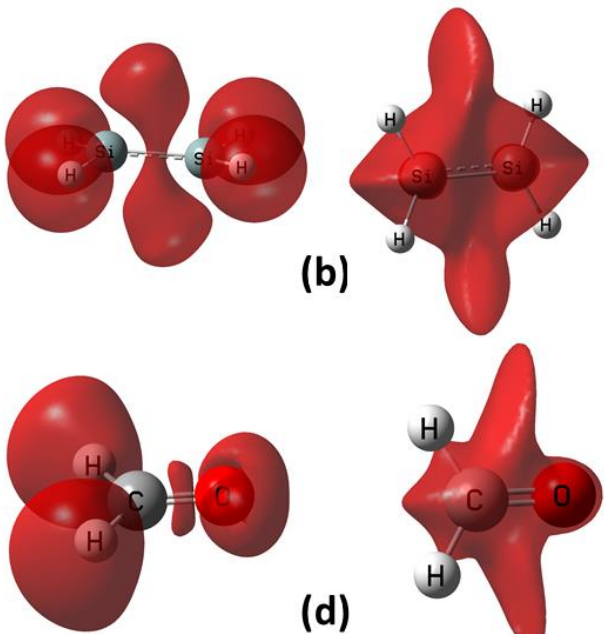

(d)

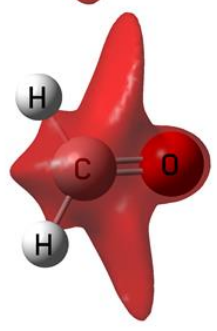

Figure 1. Comparing the results from two formulas, SCI [left, Eq. (4)] and NBI [right, Eq. (6)], with double covalent bonds for (a) $\mathrm{C}_{2} \mathrm{H}_{4}$; (b) $\mathrm{Si}_{2} \mathrm{H}_{4}$; (c) $\mathrm{S}_{2}$ and (d) $\mathrm{HCHO}$ with the isosurface value of $\mathrm{NBI}$ equal to $0.65,0.65,0.65$ and 0.8 a.u., respectively, and that of $\mathrm{SCl}$ equal to 2.0 a.u. 


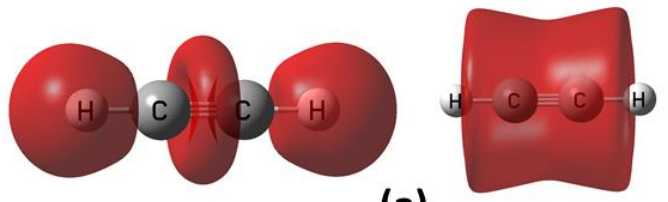

(a)

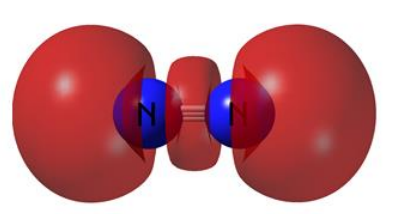

(c)

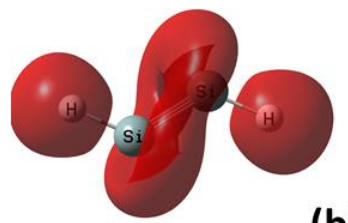

(b)
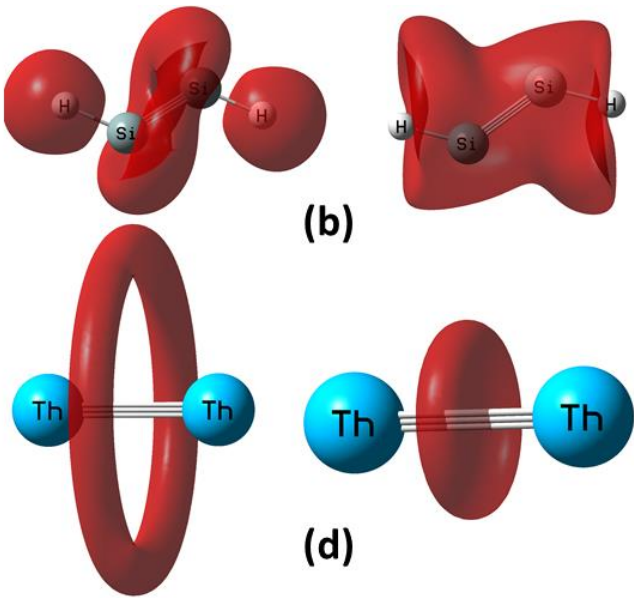

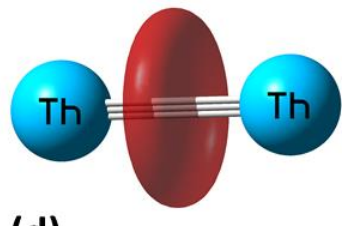

(d)

Figure 2. Comparing the results from two formulas, $\mathrm{SCl}$ (left) and $\mathrm{NBI}$ (right), with triple covalent bonds for (a) ethylene; (b) $\mathrm{Si}_{2} \mathrm{H}_{4}$; (c) $\mathrm{N}_{2}$ and (d) $\mathrm{Th}_{2}{ }^{2+}$. Their isosurface value is $0.2,0.3,0.15$ and 3.5 a.u., respectively, for $\mathrm{NBI}$ and 2.0, 2.0, 1.7, and 2.0 a.u. for $\mathrm{SCl}$. 


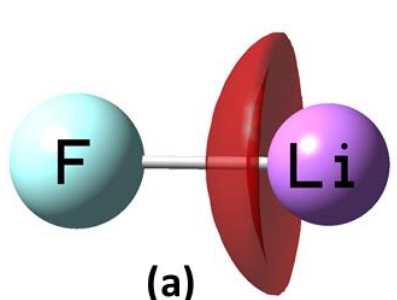

(a)

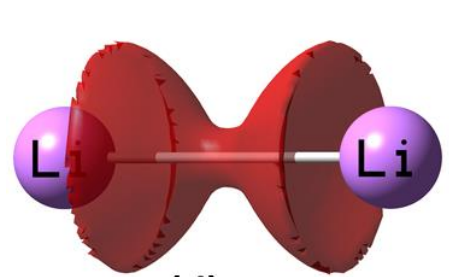

(d)

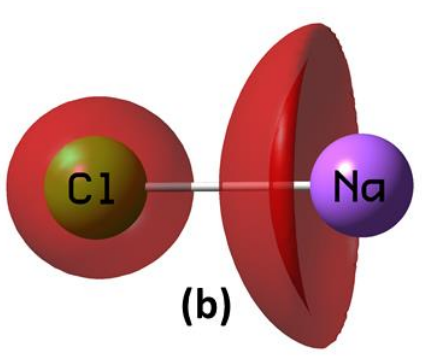

(b)

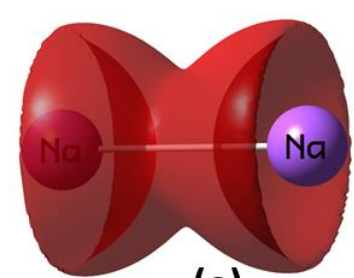

(e)
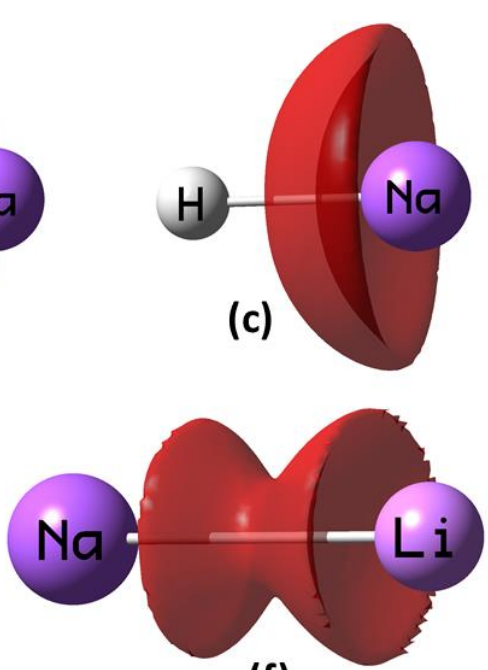

(f)

Figure 3. Using NBI to describe ionic and metallic bonds for (a) LiF; (b) $\mathrm{NaCl}$; (c) NaH; (d) $\mathrm{Li}_{2} ;$ (e) $\mathrm{Na}_{2}$ and (f) NaLi with the isovalue equal to $1.5,1.0,1.0,6.5,2.0$ and 6.5 a.u., respectively. 


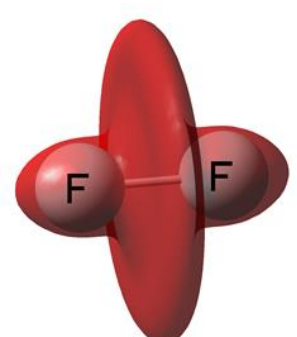

(a)

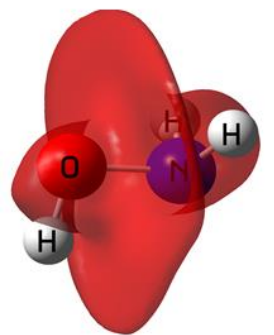

(e)

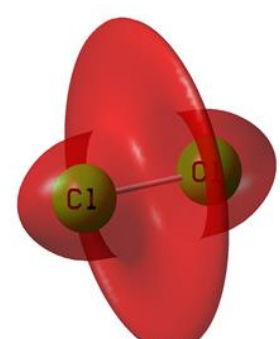

(b)

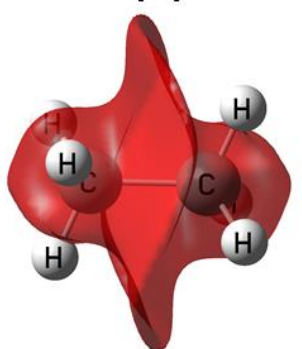

(f)

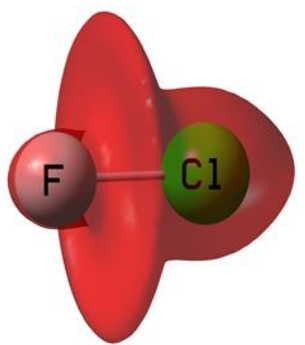

(c)

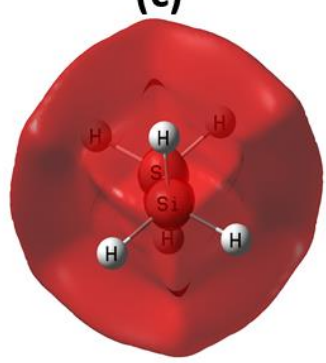

(g)

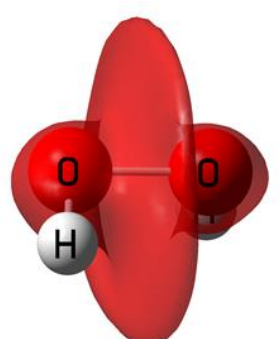

(d)

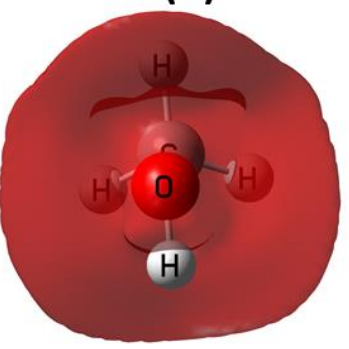

(h)

Figure 4. Using NBI to describe single covalent bond for (a) $\mathrm{F}_{2}$; (b) $\mathrm{Cl}_{2}$; (c) $\mathrm{FCl}$; (d) $\mathrm{H}_{2} \mathrm{O}_{2}$; (e) $\mathrm{NH}_{2} \mathrm{OH}$; (f) $\mathrm{C}_{2} \mathrm{H}_{6}$; (g) $\mathrm{Si}_{2} \mathrm{H}_{6}$ and (h) $\mathrm{CH}_{3} \mathrm{OH}$ with their isovalue equal to 0.6, 0.6, 0.8, 0.8, 0.65, 0.8, 0.6 and 0.65 a.u., respectively. 


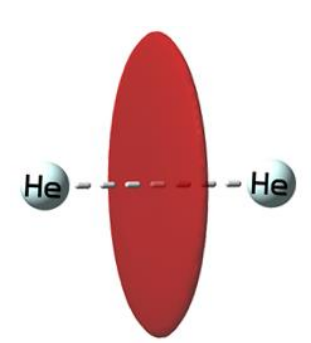

(a)

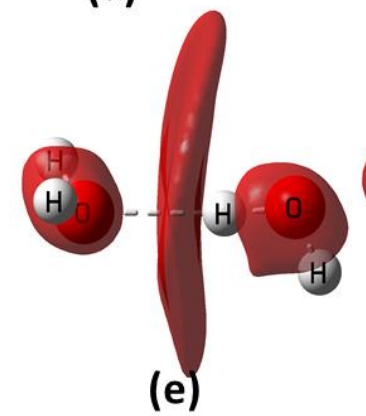

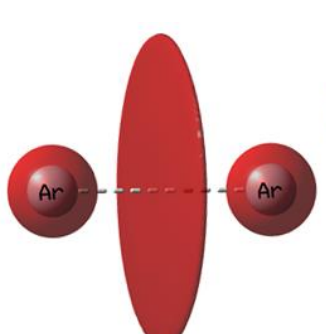

(b)

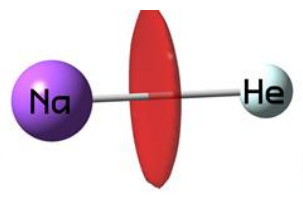

(c)
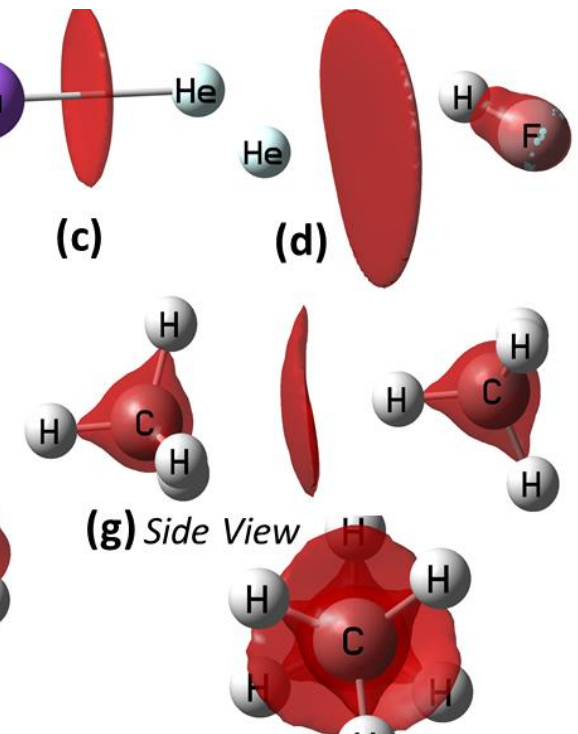

(g) Top View

Figure 5. Using NBI to describe noncovalent interactions including dispersion, charge and dipole interactions, and hydrogen bonding for (a) He dimer; (b) Ar dimer; (c) $\mathrm{He}$ and $\mathrm{Na}^{+}$diatom; (d) HF and He complex; (e) water dimer; (f) HF dimer and (g) methane dimer. Their isovalue is $0.6,0.6$, $1.5,1.0,0.6,0.6$, and 4.0 a.u., respectively. 


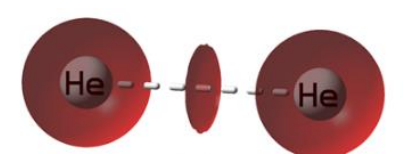

(a)

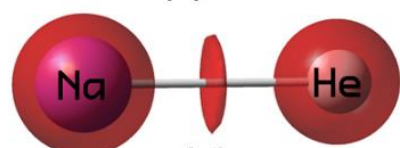

(d)

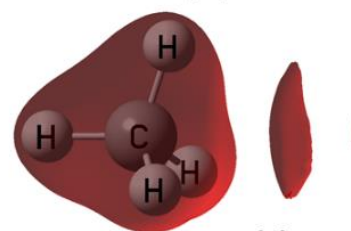

(f)

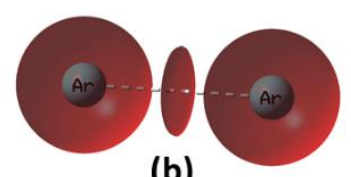

(b)

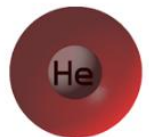

(c)

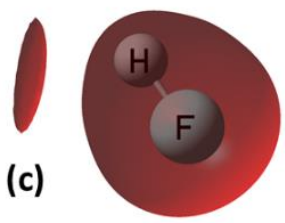

(e)
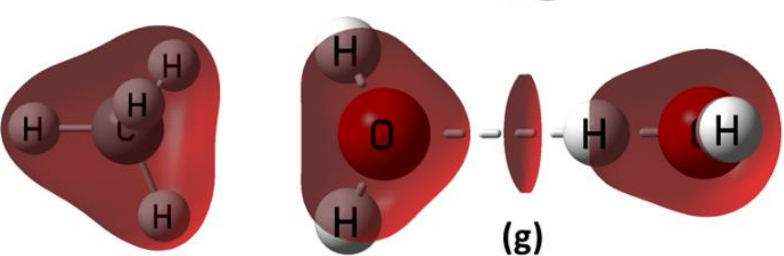

(g)

Figure 6. Signature isosurfaces from the NCl index, Eq. (7), for (a) He dimer; (b) Ar dimer; (c) HF He complex; (d) $\mathrm{Na}^{+}$and He diatom; (e) HF dimer; (f) methane dimer and (g) water dimer. Their isovalue is $10.0,10.0,10.0,8.0,5.0,6.0$, and 5.0 a.u., respectively. 


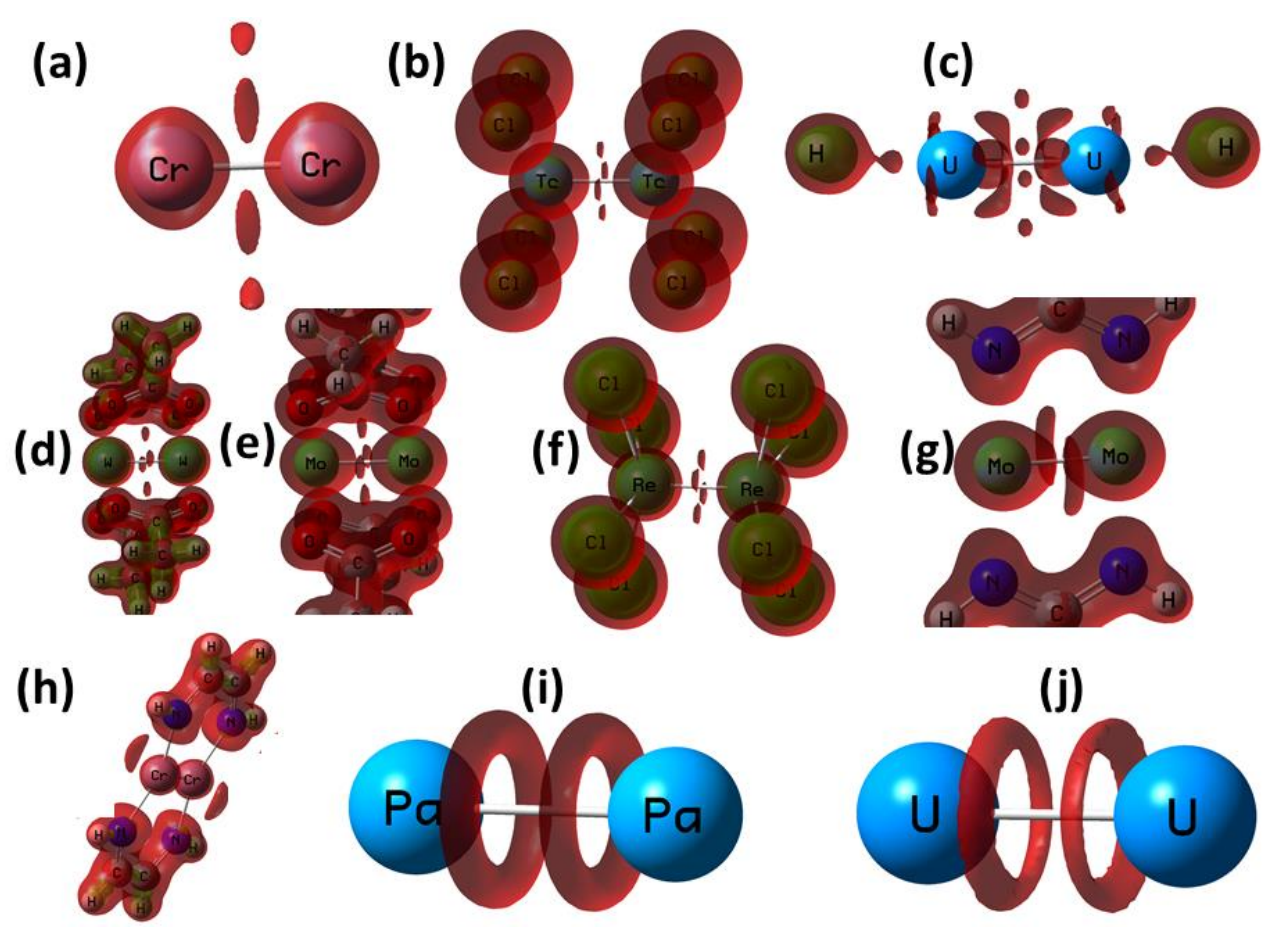

Figure 7. Signature isosurfaces from the USI index, Eq. (8), for systems with quadruple (a-f), quintuple (g-h), and sextuple (i-j) covalent bonds: (a) $\mathrm{Cr}_{2}{ }^{2+}$; (b) $\mathrm{Tc}_{2} \mathrm{Cl}_{8}{ }^{2-}$; (c) $\mathrm{U}_{2} \mathrm{H}_{2}$; (d) $\mathrm{W}_{2}\left(\mathrm{CH}_{3} \mathrm{CO}_{2}\right)_{4}$; (e) $\mathrm{Mo}_{2}\left(\mathrm{CH}_{3} \mathrm{CO}_{2}\right)_{6}$; (f) $\mathrm{Re}_{2} \mathrm{Cl}_{8}{ }^{2-}$; (g) dimolybdenum complex with two diamido bridging ligands; (h) dichromium complex with diazadiene bridging ligands; (i) $\mathrm{Pa}_{2}$; and (j) $\mathrm{U}_{2}{ }^{2+}$ with their isovalue to be $11.0,8.5,3.5,4.0,8.0,5.0,9.0,6.0,3.5$ and 3.5 a.u., respectively. 


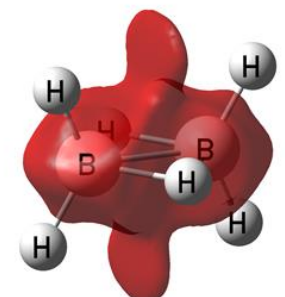

(a) $\mathrm{B}_{2} \mathrm{H}_{6}$

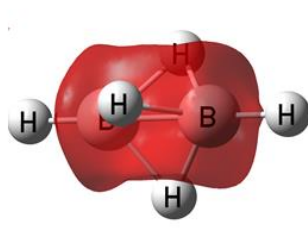

(e) $\mathrm{B}_{2} \mathrm{H}_{5}{ }^{+}$

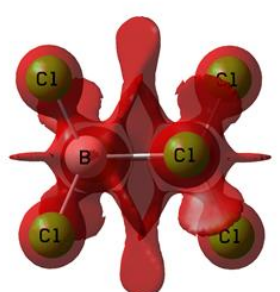

(b) $\mathrm{B}_{2} \mathrm{Cl}_{6}$

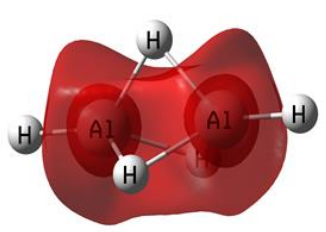

(f) $\mathrm{Al}_{2} \mathrm{H}_{5}{ }^{+}$

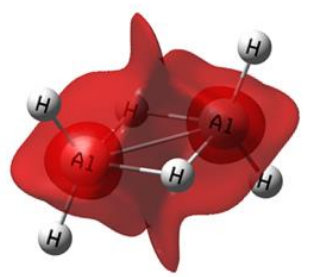

(c) $\mathrm{Al}_{2} \mathrm{H}_{6}$

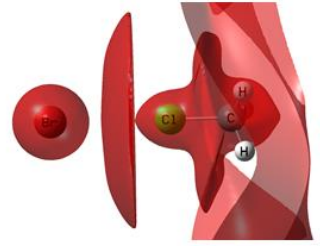

(d) $\mathrm{Al}_{2} \mathrm{Cl}_{6}$

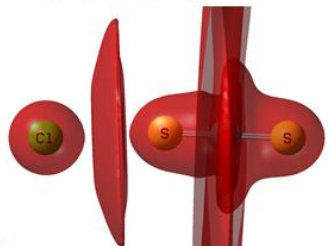

(g) Halogen Bond (h) Chalcogen Bond

Figure 8. Applying NBI index to describe systems with special bonding types, including systems with two 3c-2e bonds for (a) $\mathrm{B}_{2} \mathrm{H}_{6}$; (b) $\mathrm{B}_{2} \mathrm{Cl}_{6}$; (c) $\mathrm{Al}_{2} \mathrm{H}_{6}$; and (d) $\mathrm{Al}_{2} \mathrm{Cl}_{6}$, with three $3 \mathrm{c}-2 \mathrm{e}$ bonds for (e) $\mathrm{B}_{2} \mathrm{H}_{5}{ }^{+}$ and (f) $\mathrm{Al}_{2} \mathrm{H}_{5}{ }^{+}$, with halogen bond for $(\mathrm{g}) \mathrm{CH}_{3} \mathrm{Cl} \ldots \mathrm{Br}^{-}$and with chalcogen bond for (h) $\mathrm{S}=\mathrm{C}=\mathrm{S} \ldots \mathrm{Cl}^{-}$. Their isosurface value is equal to $1.5,3.2,2.5,1.0,1.0,1.5,0.55$ and 0.55 a.u., respectively. 


\section{TOC Graphic}

$$
\begin{array}{rr}
\mathrm{NCI} \equiv \frac{|\nabla \rho(r)|}{\rho^{4 / 3}(r)} & \text { USI } \equiv \frac{\nabla^{2} \rho(r)}{\rho^{5 / 3}(r)} \\
\mathrm{SCl} \equiv \frac{\tau_{T F}(r)}{\tau(r)-\tau_{W}(r)} & \mathrm{NBI} \equiv \frac{\tau(r)-\tau_{W}(r)}{\tau_{W}(r)}
\end{array}
$$

